\title{
Exploração de fatores de risco para lesões desportivas em atletas de tênis de mesa
}

\author{
Exploration of risk factors for sports injuries in athletes of table tennis \\ Tamlyn Shimazaki', Eliezer de Almeida', Franciele Marques Vanderlei², Dino de Aguiar Cintra Filho, \\ Luiz Carlos Marques Vanderlei ${ }^{4}$, Carlos Marcelo Pastre ${ }^{4}$, Fabio Nascimento Bastos ${ }^{5}$
}

\begin{abstract}
RESUMO I A prática do tênis de mesa requer inúmeras ações dinâmicas que podem conduzir a lesões desportivas, por isso é de importância conhecer fatores inerentes ao traumatismo nos atletas para posterior formulação dos modelos preventivos. Objetivou-se explorar os fatores de risco para lesões desportivas em mesa-tenistas. Para isso, foram entrevistados 111 atletas participantes do Campeonato Paulista de Tênis de Mesa, com média de idade de 22,39 $\pm 8,88$ anos de ambos os gêneros, recrutados ao acaso, classificados em dois níveis competitivos: regional/estadual e nacional/internacional. Utilizou-se o Inquérito de Morbidade Referida adaptado com as características do tênis de mesa com a finalidade de reunir dados pessoais, de treinamento e da lesão desportiva. Foram observadas 0,51 lesões por atleta, e os atletas de nível nacional/internacional apresentaram maiores índices de lesão (52,94\%) do que os de nível estadual/regional (48,84\%). No gesto específico, notou-se que os membros superiores (93,62\%) e o tronco (87,5\%) são os locais mais acometidos. Para ambos os níveis, o treinamento foi o momento mais relatado de ocorrência dos agravos. Conclui-se que atletas de nível nacional/internacional possuem maiores índices de lesão e que o gesto específico é a principal causa das lesões, acometendo principalmente os membros superiores e o tronco e ocorrendo com maior frequência durante o treinamento
\end{abstract}

Descritores I traumatismos em atletas; fatores de risco; inquéritos de morbidade.
ABSTRACT I The practice of table tennis requires numerous dynamic actions which could conduce to athletic injuries. Due to the shortage of studies on this modality, there is important to know inherent factors to athletic injuries for a preventive patterns subsequent formulation. The objective of this study was to explore the risk factors to athletic injuries in table tennis players. For that, there were evaluated 111 athletes who participated of Paulista Championship of Table Tennis, with mean age of $22.39 \pm 8.88$ years, of both gender, recruited on a randomized way and classified into two level of competition: regional/state and national/international. It was used the Reported Morbidity Survey adapted to the table tennis characteristics in order to gathering information about the personal data, training data and athletic injuries. There was observed 0.51 injuries by athlete, and the athletes of national/international level presented the higher indexes of injury (52.94\%) when compared with regional/state (48.84\%). On specific gesture, the upper limbs (93.62\%) and the trunk (87.5\%) were the most affected sites. For both level of competition, the training was the more related moment of the injuries occurrence. The conclusions are that the athletes of national/international level present higher indexes of injury and the specific gesture is the principal reason of injury, affecting mainly the upper limbs and trunk, occurring more frequently during the training.

Keywords I athletic injuries; risk factors; morbidity surveys.

Estudo desenvolvido na cidade de Presidente Prudente (SP), Brasil.

'Graduados em Educação Física pela Universidade Estadual Paulista "Júlio de Mesquita Filho" (UNESP) - Presidente Prudente (SP), Brasil. 2Doutoranda em Medicina (Cardiologia) pela Universidade Federal de São Paulo (UNIFESP) - São Paulo (SP), Brasil.

${ }^{3}$ Docente da Faculdade de Ciências e Tecnologia da UNESP - Presidente Prudente (SP), Brasil.

${ }^{4}$ Docente em Fisioterapia da UNESP. Faculdade de Ciências e Tecnologia - Presidente Prudente (SP), Brasil.

${ }^{5}$ Docente da Universidade Estadual do Norte do Paraná (UENP) - Jacarezinho (PR), Brasil. 


\section{INTRODUÇÃO}

O tênis de mesa, originado na Inglaterra por volta do final do século XIX, é uma das modalidades esportivas mais praticadas no mundo ${ }^{1,2}$. No Brasil, sua prática teve início com turistas ingleses em $1905^{1}$ e, atualmente, por meio da Confederação Brasileira de Tênis de Mesa (CBTM), essa modalidade está organizada em todos os estados do País e tem obtido grandes resultados em competições na América Latina ${ }^{3}$.

Os bons resultados obtidos fazem aumentar ainda mais a procura da população por essa modalidade, seja como forma de lazer ou competição. A busca de resultados no âmbito competitivo impõe altas cargas de treinamentos, incluindo treinos de força, resistência, agilidade, técnico e tático, os quais podem produzir lesões por sobrecarga, além das inerentes à própria prática esportiva ${ }^{2}$.

Apesar de esse esporte não ter contato físico entre os atletas e ser considerado de pouco impacto em comparação com outros esportes que utilizam raquetes, como o tênis e o badminton, Petri et al. ${ }^{2}$ descrevem a ocorrência de lesões com a prática do tênis de mesa, pois para o atleta ter um bom desempenho em competições de alto nível é necessário um programa de treinamento intenso que pode chegar a oito horas diárias, dependendo do indivíduo.

O único estudo realizado no Brasil que descreve as lesões desportivas nos mesa-tenistas foi de Petri et al. ${ }^{2}$. Neste estudo, foi avaliada a correlação entre lesões musculoesqueléticas com a carga e o tipo de treinamento de 116 jogadores de tênis de mesa e foi observada baixa incidência de lesão, as quais ocorreram principalmente no tornozelo, joelho e punho. Em âmbito internacional, os estudos abordam questões específicas do gesto esportivo, como análise dos movimentos realizados com a raquete $^{4}$ e velocidade de reação com a bola ${ }^{5}$, no entanto não foram encontrados estudos que abordam exclusivamente as lesões desportivas nesses jogadores.

É de conhecimento de muitos pesquisadores, técnicos e atletas a importância do mapeamento detalhado no que diz respeito às modalidades esportivas, assim como com relação aos benefícios e malefícios que estas podem proporcionar, além da identificação da frequência, chances e formas de adquirir e prevenir as diversas lesões a que os atletas estão sujeitos, tanto em treinos como em competições ${ }^{1}$.

Entendendo como relevante a necessidade de $\mathrm{co}^{-}$ nhecer fatores inerentes ao traumatismo em atletas praticantes de tênis de mesa para posterior formulação de modelos preventivos e de treinamento, e em decorrência da escassez de estudos realizados nesta modalidade, torna-se de fundamental importância a realização do presente estudo. Assim, constituiu-se como objetivo explorar os fatores de risco para lesões desportivas em mesa-tenistas a partir da aplicação do Inquérito de Morbidade Referida (IMR).

\section{MATERIAIS E MÉTODOS}

\section{Natureza e população do estudo}

O estudo caracteriza-se como observacional e analítico. Participaram deste estudo 111 atletas participantes do Campeonato Paulista de Tênis de Mesa no ano de 2010, ocorrido na cidade de Presidente Prudente, São Paulo, sendo 73 do gênero masculino e 38 do gênero feminino, com média de idade de 22,39 $\pm 8,88$ anos, massa corporal de $65,77 \pm 12,37 \mathrm{~kg}$, estatura de 1,69 $\pm 0,08 \mathrm{~m}$, tempo de treinamento de 9,21 $\pm 7,30$ anos e horas semanais de prática esportiva de $13,90 \pm 9,87$ horas. A presente pesquisa foi apreciada e aprovada pelo Comitê de Ética em Pesquisa da Faculdade de Ciências e Tecnologia (FCT/UNESP) campus de Presidente Prudente/SP, sob o Protocolo no 28/2010.

\section{Técnicas e procedimentos de campo}

Os dados obtidos foram coletados a partir de entrevistas por meio de um IMR modificado, o qual é um instrumento bastante utilizado para obter informações sobre o estado de saúde de uma população específica, em razão de sua aplicabilidade e objetividade ${ }^{6}$, com solicitações das informações retroativas à temporada corrente, ou seja, aproximadamente 12 meses. Apesar disso, esse inquérito não foi validado para a população estudada, o que representa uma limitação do estudo. Foram incluídos no estudo os atletas que praticassem a modalidade há mais de um ano e que participaram de competições durante a temporada corrente (Apêndice).

Os atletas foram entrevistados no local da competição em um momento no qual não estavam competindo, a fim de não interferir na dinâmica e concentração da competição. Previamente, houve uma explicação sobre a realização e a finalidade da pesquisa para então o sujeito assinar o termo de consentimento livre e esclarecido. As perguntas do IMR, o qual pode trazer informações sobre quaisquer lesões que o atleta tenha sofrido 
durante a prática da modalidade, foram realizadas pelos pesquisadores, respondidas pelos próprios atletas e anotadas em fichas individualizadas ${ }^{6}$.

Para garantir a fidedignidade do estudo, foi realizado inicialmente um estudo piloto para ajustar os procedimentos metodológicos e testar a ferramenta diante de uma população envolvida no tênis de mesa, com nível de desempenho semelhante ao da amostra do presente estudo. Tal procedimento foi considerado importante para aperfeiçoar o instrumento, além de permitir maior contato do entrevistador com a metodologia em uso, evitando, assim, possíveis falhas durante a coleta de dado. Ressalta-se que a coleta foi realizada por dois entrevistadores previamente treinados.

Por fim, as fichas de coletas foram numeradas para facilitar o registro das informações, e os dados foram registrados em planilha computacional para organização, sistematização e análise dos dados.

\section{Inquérito de morbidade referida e variáveis envolvidas}

O IMR foi adaptado para as características do tênis de mesa a partir de um modelo fechado descrito por Pastre et al. ${ }^{6}$, contendo dados pessoais e de treinamento dos atletas, como: gênero, idade, massa corporal, estatura, nível de performance (estadual, regional, nacional ou internacional), tempo de treinamento em anos e número de horas semanais de treino. Além disso, foram coletadas informações sobre as lesões, abordando o local anatômico, mecanismo e momento em que ocorreu o agravo.

Para o local anatômico acometido, referente ao sintoma de dor ou desconforto musculoesquelético relatado pelo voluntário, uma figura ilustrativa do corpo humano foi mostrada ao atleta, com a finalidade de facilitar a identificação por parte deste. Dessa forma, o sujeito poderia assinalar no próprio desenho o local da lesão. Em relação ao mecanismo de lesão, foi considerado como sendo a condição causadora do agravo (exercícios aeróbicos, exercícios de força, movimentação de pernas e gestos específicos), caracterizado pela percepção do atleta sobre o momento exato em que surgiram os sinais e sintomas típicos de episódio agudo e/ou o tipo de atividade em que tais manifestações se acentuaram. Já o momento de ocorrência da lesão visou observar se a lesão aconteceu durante a fase específica de treinamento ou competição.

No presente estudo, foi considerada uma lesão desportiva qualquer comprometimento do sistema musculoesquelético, cuja instalação de sinais e sintomas fosse provenientes da prática esportiva, em fase de treinamento ou competição, que tenha comprometido o seu treinamento normal, seja na forma, duração, intensidade ou frequência, conforme já utilizado em estudos anteriores $^{6,7}$.

\section{Organização e descrição das categorias das variáveis}

Para facilitar a análise e apresentação dos resultados, as categorias ou subdivisões das variáveis foram agrupadas, sem, no entanto, modificar a essência de sua origem ou as conclusões do estudo.

A variável "nível de competitividade" foi dividida em duas categorias: (1) nível estadual/regional em que os atletas participavam de competições, como campeonatos do estado ou da região; e (2) nível nacional/internacional em que os atletas participavam de campeonatos brasileiros ou internacional. O mecanismo de lesão foi organizado em quatro tipos de categorias: exercícios aeróbicos, exercícios de força, movimentação de pernas e gestos específicos, de acordo com as principais características dos movimentos exigidos no tênis de mesa, como forehand/backhand, drive, topspin, smash, flick, slice e saques. Por fim, para localização anatômica, os seguintes segmentos foram escolhidos para análise dos dados: tronco, membros superiores (MMSS) e membros inferiores (MMII).

\section{Análise estatística}

A estatística descritiva foi realizada por meio da tendência central de variabilidade (média e desvio padrão). A distribuição da normalidade dos dados foi testada utilizando o teste de Kolmogorov-Smirnov. Em relação às variáveis quantitativas, foi analisada sua relação com a presença ou ausência de lesão. Dessa forma, as variáveis paramétricas foram testadas a partir do teste $t$ de Student; já as que não apresentaram distribuição Gaussiana, foram testadas por meio do teste de Mann-Whitney.

O estudo da associação entre as variáveis qualitativas envolvendo as características da lesão e do esporte foi realizado utilizando o teste de Goodman para contrastes entre e dentro de populações multinomiais. Para a comparação entre os gêneros, foi utilizado teste de Odds Ratio com intervalo de confiança de $95 \%$. Todas as conclusões foram descritas no nível 5\% de significância. 


\section{RESULTADOS}

A distribuição de frequência de lesão de acordo com o gênero não mostrou diferença estatisticamente significante (OD=0,57; IC95\% 0,26-1,26), por isso as análises foram feitas sem distinção de gênero. Entre os mesa-tenistas, houve um índice de lesão de 0,51 lesão por atleta. Notase que não há diferença para as variáveis antropométricas e de treinamento (Tabela 1).

Tabela 1. Medidas descritivas das variáveis antropométricas e de treinamento de acordo com a presença ou ausência de lesão

\begin{tabular}{|c|c|c|c|}
\hline \multirow[b]{2}{*}{ Variáveis } & \multicolumn{3}{|c|}{ Lesão } \\
\hline & $\begin{array}{l}\text { Presente } \\
(n=57)\end{array}$ & $\begin{array}{c}\text { Ausente } \\
(n=54)\end{array}$ & Valor $p$ \\
\hline Idade (anos) ${ }^{b}$ & $\begin{array}{c}22,81 \pm 8,98 \\
(18,00)\end{array}$ & $\begin{array}{c}21,96 \pm 8,85 \\
(18,50)\end{array}$ & 0,41 \\
\hline Massa corporal (kg) & $\begin{array}{c}64,43 \pm 11,97 \\
(63,00)\end{array}$ & $\begin{array}{c}67,20 \pm 12,74 \\
(68,00)\end{array}$ & 0,23 \\
\hline Estatura $(\mathrm{m})^{\mathrm{a}}$ & $\begin{array}{c}1,68 \pm 0,08 \\
(1,68)\end{array}$ & $\begin{array}{c}1,70 \pm 0,09 \\
(1,70)\end{array}$ & 0,33 \\
\hline IMC $\left(\mathrm{kg} / \mathrm{m}^{2}\right)^{\mathrm{a}}$ & $\begin{array}{c}22,55 \pm 3,11 \\
(22,06)\end{array}$ & $\begin{array}{c}23,08 \pm 3,25 \\
(22,46)\end{array}$ & 0,37 \\
\hline Tempo de treino (anos) ${ }^{b}$ & $\begin{array}{c}9,36 \pm 7,06 \\
(7,00)\end{array}$ & $\begin{array}{c}9,05 \pm 7,62 \\
(6,00)\end{array}$ & 0,47 \\
\hline Horas semanais’ & $\begin{array}{c}14,52 \pm 10,19 \\
(12,00)\end{array}$ & $\begin{array}{c}13,14 \pm 9,72 \\
(13,00)\end{array}$ & 0,50 \\
\hline
\end{tabular}

Teste de normalidade de Kolmogorov-Smirnov

aTeste $t$ de Student

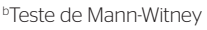

Não foi observada diferença significante entre os níveis de competitividade analisados. Do total de 43 mesa-tenistas inseridos no nível estadual/regional, 21 $(48,84 \%)$ apresentaram lesões desportivas, enquanto no nível nacional/internacional, dos 68 mesa-tenistas analisados, $36(56,94 \%)$ apresentaram agravos musculoesqueléticos.

Em relação ao mecanismo causal, observa-se na Tabela 2 que houve diferença significativa entre os níveis de competitividade. Atletas de nível nacional/internacional apresentaram maior índice de lesões em exercícios aeróbicos $(10,29 \%)$ do que os de nível estadual/ regional. Para ambos os grupos, nota-se que o gesto específico é o principal fator causal das lesões. Em relação ao momento da lesão, pode-se observar que mesa-tenistas tanto de nível estadual/regional como nacional/ internacional são mais acometidos por lesão durante o treinamento (Tabela 2).

A Tabela 3 mostra a distribuição de frequência de lesões do mecanismo causal segundo local anatônico acometido. No gesto específico, nota-se que o tronco $(87,5 \%)$ e os MMSS $(93,62 \%)$ são os locais mais acometidos por lesões em relação aos MMII (16,13\%). Comparando os MMII aos mecanismos de lesão, observa-se predominância na movimentação de pernas com $64,52 \%$ das lesões em relação ao gesto específico com $16,13 \%$ (Tabela 3).

Tabela 2. Distribuição de frequência, absoluta (n) e relativa (\%), da variável mecanismo e momento de lesão segundo nível de competitividade em mesa-tenistas

\begin{tabular}{|c|c|c|c|c|c|}
\hline \multirow[b]{2}{*}{ Nível } & \multicolumn{4}{|c|}{ Mecanismo de lesão } & \multirow[b]{2}{*}{ Total } \\
\hline & $\begin{array}{l}\text { Exercícios } \\
\text { aeróbicos }\end{array}$ & $\begin{array}{l}\text { Exercícios } \\
\text { de força }\end{array}$ & $\begin{array}{l}\text { Movimentação } \\
\text { de pernas }\end{array}$ & $\begin{array}{c}\text { Gesto } \\
\text { específico }\end{array}$ & \\
\hline Estadual/Regional & $\mathrm{O}(\mathrm{O}, \mathrm{OO})$ & $\mathrm{O}(\mathrm{O}, \mathrm{OO})$ & $9(26,47)^{*}$ & $25(73,53)^{\dagger}$ & $34(100)$ \\
\hline Nacional/Internacional & $7(10,29)^{\ddagger}$ & $3(4,41)$ & $13(19,12)$ & $45(66,18)^{\dagger}$ & $68(100)$ \\
\hline \multirow{2}{*}{ Nível } & \multicolumn{4}{|c|}{ Momento da lesão } & \multirow{2}{*}{ Total } \\
\hline & \multicolumn{2}{|c|}{ Treinamento } & \multicolumn{2}{|c|}{ Competição } & \\
\hline Estadual/Regional & \multicolumn{2}{|c|}{$28(82,35)^{\#}$} & \multicolumn{2}{|c|}{$6(17,65)$} & $34(100)$ \\
\hline Nacional/Internacional & \multicolumn{2}{|c|}{$59(86,76)^{\#}$} & \multicolumn{2}{|c|}{$9(13,24)$} & $68(100)$ \\
\hline
\end{tabular}

Teste de Goodman para contrastes entre e dentro de populações multinomiais; *Diferença em relação aos mecanismos de exercícios aeróbicos, exercícios de força e gesto específico; ‘Diferença em relação aos mecanismos de exercícios aeróbicos, exercícios de força e movimentação de pernas; *Diferença em relação ao nível estadual/regional; "diferença em relação ao momento competição.

Tabela 3. Distribuição de frequência, absoluta (n) e relativa (\%), da variável mecanismo da lesão segundo local anatômico acometido em mesa-tenistas

\begin{tabular}{|c|c|c|c|c|c|}
\hline \multirow[b]{2}{*}{ Local anatômico } & \multicolumn{4}{|c|}{ Mecanismo de lesão } & \multirow[b]{2}{*}{ Total } \\
\hline & $\begin{array}{l}\text { Exercícios } \\
\text { aeróbicos }\end{array}$ & $\begin{array}{l}\text { Exercícios } \\
\text { de força }\end{array}$ & $\begin{array}{l}\text { Movimentação } \\
\text { de pernas }\end{array}$ & $\begin{array}{c}\text { Gesto } \\
\text { específico }\end{array}$ & \\
\hline Tronco & $1(4,17)$ & $\mathrm{O}(0,00)$ & $2(8,33)$ & $21(87,5)^{*}$ & $24(100)$ \\
\hline MMSS & $\mathrm{O}(0,00)$ & $3(6,38)$ & $\mathrm{O}(0,00)$ & $44(93,62)^{\star}$ & $47(100)$ \\
\hline MMII & $6(19,35)$ & $\mathrm{O}(0,00)$ & $20(64,52)^{\ddagger \ddagger}$ & $5(16,13)^{\ddagger}$ & $31(100)$ \\
\hline
\end{tabular}

Teste de Goodman para contrastes entre e dentro de populações multinomiais; * Diferença em relação ao mecanismo de exercícios aeróbicos, exercícios de força e movimentação das pernas; ‘Diferença em relação ao mecanismo de exercícios aeróbicos, exercícios de força e gesto específico; ‘ 'Diferença em relação ao tronco e MMSS; MMSS: membros superiores; MMIl: membros inferiores. 


\section{DISCUSSÃO}

O presente estudo buscou explorar os fatores de risco para lesões desportivas em mesa-tenistas, associando-as a fatores de risco específicos da modalidade e do atleta a partir da aplicação do IMR.

Os resultados mostraram que a frequência de lesão foi maior para jogares de nível nacional/internacional $(52,94 \%)$ em comparação aos de nível estadual/regional $(48,84 \%)$. A diferença na frequência de lesão entre os diferentes níveis é pequena, no entanto deve ser considerada. Uma possível hipótese se refere a jogadores de menor nível de competitividade treinar em menor intensidade comparada aos atletas de alto nível que possuem um treinamento mais intenso para melhorar o desempenho técnico e tático durante as diferentes situações de jogo ${ }^{1}$.

O índice de lesão foi de 0,51 lesão por atleta, caracterizando-se como baixo comparado com outros esportes como ginástica artística ${ }^{7}$, futebol $^{8}$ e rugby ${ }^{9}$. Ressalta-se que esportes com raquete apresentam baixa frequência de lesões. Tal fato pode estar relacionado à ausência de contato físico, sendo este considerado um dos grandes fatores predisponentes a traumas ${ }^{2}$. Por outro lado, não se deve descartar a ocorrência das lesões em esportes que não apresentam impacto direto com o oponente.

Durante uma partida de tênis de mesa, as bolas são enviadas em diferentes direções com velocidades e rotações igualmente variáveis, fazendo que os competidores realizem movimentos complexos em frações de segundo, envolvendo essencialmente deslocamento lateral e pequenos saltos com mudanças de direções que deixam os atletas expostos às lesões ${ }^{1,2}$.

Em relação às variáveis antropométricas, não foi observada diferença significante quando comparadas presença e ausência de lesão, corroborando os achados de Petri et al. ${ }^{2}$, que descrevem não haver diferença entre idade, gênero, etnia, estilo de jogo e forma de segurar a raquete. A mesma condição é evidenciada por Smekal et al. ${ }^{10}$, que relata não haver necessidade de um biótipo especial para a prática do tênis de mesa.

No presente estudo, não houve diferença significativa para as variáveis tempo de treinamento e horas semanais de treino, divergindo dos achados de Petri et al. ${ }^{2}$, que descrevem o número de horas de treino por semana como fator de risco para a instalação de lesões. As diferentes características entre os sujeitos estão relacionadas na variação da angulação dos movimentos técnicos. Destaca-se o mesa-tenista com vasto repertório de movimentos e variações técnicas e táticas durante o jogo, além dos que apresentarem melhor condição física ${ }^{1}$.
Sobre o mecanismo causal, foi verificado que atletas de nível nacional/internacional apresentaram maior frequência de lesão em exercícios aeróbicos (10,29\%) do que os de nível estadual/regional (0,00\%). Como os atletas de alto nível procuram um melhor desempenho durante o jogo e um fator contribuinte para isso é uma boa aptidão aeróbica ${ }^{1}$, altas cargas de treinamento, entendidas por elevado volume e intensidade de treinos, podem possibilitar maiores índices de lesão nesses praticantes $^{1,2}$.

O principal fator causal de lesões mostrou ser os gestos específicos comparando com exercícios aeróbicos, exercícios de força e movimentação de pernas, possivelmente em decorrência do excesso de repetição e intenso volume de treinamento ${ }^{1,10}$. A atividade de um jogador de tênis de mesa é caracterizada pela alta complexidade das técnicas de coordenação, rápido ritmo, qualidade acumulativa e explosiva de esforço físico, alta precisão e um amplo ponto de mira, para que a colocação das bolas seja realizada por toda área de jogo ${ }^{1,11}$.

Portanto, é necessário aprender e aperfeiçoar os movimentos específicos da modalidade, além de conseguir utilizar toda habilidade em diversas situações de jogo com modificações durante a partida, diferentes níveis técnicos e estilos dos adversários. Para isso, é necessário um intenso volume de trabalho e muitas repetições de movimentos para corrigir erros na movimentação e também para que o atleta adquira confiança na colocação dos golpes ${ }^{1}$.

Entre os mesa-tenistas, os locais mais acometidos foram tronco e MMSS, condições estas já observadas por Ellwood ${ }^{12}$ e DiCarlo et al. ${ }^{13}$ que notaram altos valores de bolas rebatidas durante uma partida, caracterizando maior número de ações nos MMSS e tronco. Por outro lado, Petri et al. ${ }^{2}$ relataram maior frequência de lesões nos MMII, tal fato relacionado à grande quantidade de deslocamentos laterais que o esporte exige.

Atletas participantes dos níveis estadual/regional e nacional/internacional apresentam maior frequência de lesão no momento treinamento. Tal condição pode estar relacionada à maior exposição destes nesse momento quando comparado às competições. Segundo Petri et al. $^{2}$, o risco de lesão pode estar relacionado com a alta carga de treinamento. Treinadores e atletas buscam constantemente a melhor performance e, por sua vez, extrapolam a fase de adaptação, resultando em exaustão e contribuindo para instalação de lesões ${ }^{14}$.

Yang $^{15}$ identificou as lesões em razão de cargas excessivas aplicadas ao praticante e descrevem detalhadamente o excesso de gestos repetitivos. A possibilidade 
de interferência de fatores auxiliares pode ter relação com as repetições excessivas, destacando-se a fadiga física. Tais condições podem comprometer habilidades como força e coordenação, prejudicando o controle proprioceptivo e interferindo nos mecanismos de defesa contra agressões externas ${ }^{10}$.

Como limitação da presente pesquisa pode-se citar que o IMR utilizado como instrumento de coleta de dados não apresenta validação. No entanto, seu uso justifica-se pela necessidade de um instrumento capaz de coletar informações sobre as lesões desportivas em ambiente competitivo. Outro ponto a ser considerado é a diferença na faixa etária dos mesa-tenistas que pode ter causado influência na instalação dos agravos.

A obtenção de dados a respeito das modalidades esportivas e seus fatores de risco é de grande importância para as ciências da saúde e do esporte, uma vez que pode gerar subsídios para prevenção de lesões. Nesse sentido, a exploração de lesões e seus fatores revelam relações importantes no que tange ao local anatômico, nível de competitividade, mecanismo e momento de lesão. Portanto, espera-se que tais informações possam contribuir para melhor entendimento sobre lesões em praticantes dessa modalidade, incluindo melhor planejamento e adequação de treinamento.

Como perspectivas futuras, sugerem-se a divisão por tempo de treinamento e horas semanais de treino, além de serem analisados outros fatores de riscos que podem estar associados com a instalação de lesão, como tempo de recuperação das lesões, tipo de treinamento entre os atletas e lesões prévias.

\section{CONCLUSÃO}

Conclui-se que atletas de nível nacional/internacional apresentam maior frequência de lesão em relação aos de nível estadual/regional. Sobre o mecanismo causal, os gestos específicos revelaram ser o principal causador das lesões. O tronco e os MMSS foram os locais anatômicos mais acometidos, fatos ocorridos principalmente durante os treinamentos. Por fim, não foi constatada associação entre presença e ausência de lesão e as variáveis antropométricas e de treinamento para os mesa-tenistas.

\section{REFERÊNCIAS}

1. Marinovic W, lizuka CA, Nagaoka KT (editors). Tênis de Mesa: Teoria e Prática. São Paulo: Phorte; 2006.

2. Petri CF, Rodrigues RC, Cohen M, Abdalla RJ. Lesões músculoesqueléticas relacionadas com a prática do tênis de mesa. Rev Bras Ortop. 2002;37(8):358-62.

3. Confederação Brasileira de Tênis de Mesa. História do Tênis de Mesa: Ontem, Hoje e Amanha; 2009 [cited 2011 May 5]. Available from: http://www.cbtm.org.br/

4. Tang H, Mizoguchi M, Toyoshima S. Speed and spin characteristics of the $40 \mathrm{~mm}$ table tennis ball. Int J Table Tennis Sci. 2002;1:278-84.

5. Toriola AL, Toriola OM, Igbokwe NU. Validity of specific motor skills in predicting table-tennis performance in novice players. Percept Mot Skills. 2004;98(2):584-6.

6. Pastre CM, Carvalho Filho G, Monteiro HL, Netto Júnior J, Padovani CR. Lesões desportivas no atletismo: comparação entre informações obtidas em prontuários e inquérito de morbidade referida. Rev Bras Med Esporte. 2005;11(1):43-7.

7. Hoshi RA, Pastre CM, Vanderlei LCM, Netto Júnior J, Bastos FN. Lesões desportivas na ginástica artística: estudo a partir de morbidade referida. Rev Bras Med Esporte. 2008;14(5):440-5.

8. Lindenfeld TN, Schmitt DJ, Hendy MP, Mangine RE, Noyes FR. Incidence of injury indoor soccer. Am J Sports Med. 1994:22(3):364-71.

9. Gabbett TJ. Incidence of injury in semi-professional rugby league players. Br J Sports Med. 2003;37(1):36-44.

10. Smekal G, von Duvillard SP, Rihacek C, Pokan R, Hofmann P, Baron $\mathrm{R}$, et al. A physiological profile of tennis match play. Med Sci Sports Exerc. 2001;33(6):999-1005.

11. Nizetich $\mathrm{H}$. El papel de las caracteristicas personales del jugador de tenis de mesa em proporcionar eficiencia y estabilidad durante las competências. 1997 [cited 2011 May 18]. Available from: http://www. efdeportes.com/efd6/hen6/htm

12. Ellwood JD. Is the sub-maximal treadmill test an accurate predictor of oxygen uptake in table tennis? Int J Table Tennis Sci. 1992;1:33-40.

13. DiCarlo M, Formioni M, Peña S, Fernández FP. Biomechanics and sports shoulder lesion to table tennis. Centro Méd. 1997;42(1):18-21.

14. Allen GD. Physiological characteristics of elite Australian table tennis athletes and their responses to high level competition. J Hum Mov Studies. 1991;20(3):133-47.

15. Yang G. Funcional evaluation for table tennis players. Int J Table Tennis Sci. 1992:1:95-7. 
Apêndice. Inquérito de Morbidade Referida (IMR)
VO - Identificação:
V1 - Sexo:
V2 - Idade:
V3 - Peso:
V4 - Estatura:
V5 - Nível de performance: (1) Estadual $\quad$ (2) Regional $\quad$ (3) Nacional (4) Internacional
V6 - Tempo de treinamento:___ anos V7 - Horas por semana de treino: __ horas
V8 - Presença de lesão: (1) Sim (2) Não

\begin{tabular}{|c|c|c|c|c|c|c|c|c|c|c|}
\hline \multirow{2}{*}{ VARIÁVEL } & \multicolumn{10}{|c|}{ LESÃO } \\
\hline & 1 & 2 & 3 & 4 & 5 & 6 & 7 & 8 & 9 & 10 \\
\hline \multicolumn{11}{|l|}{ v9 - Local anatômico } \\
\hline \multicolumn{11}{|l|}{ V10 - Mecanismo de lesão } \\
\hline V11 - Momento do agravo & & & & & & & & & & \\
\hline
\end{tabular}

\begin{tabular}{|c|c|c|c|c|}
\hline \multirow[t]{2}{*}{ Variáveis } & \multicolumn{4}{|c|}{ Categoria das variáveis } \\
\hline & (1) Pescoço & (2) Ombro & (3) Braço & (4) Região dorsal \\
\hline v9 - Local anatômico & (5) Cotovelo & (6) Antebraço & (7) Região lombar & (8) Punho/Mão \\
\hline & (9) Quadril/Coxa & (10) Joelho & (11) Panturrilha & (12) Tornozelo/Pé \\
\hline V10 - Mecanismo da lesão & (1) Exercícios aeróbicos & (2) Exercícios de força & (3) Movimento de pernas & (4) Gesto específico \\
\hline V11 - Momento do agravo & (1) Treinamento & (2) Competição & & \\
\hline
\end{tabular}

